\title{
THE FIRST COHOMOLOGY GROUPS OF NEST ALGEBRAS ON NORMED SPACES
}

\author{
HAN DEGUANG
}

(Communicated by Palle E. T. Jorgensen)

\begin{abstract}
In this short note we prove that all the first cohomology groups of nest algebras acting on a normed space are trivial when the coefficients lie in any weakly closed bimodule which contains the nest algebra.
\end{abstract}

In [1] Christensen proved that all the first cohomology groups of a nest algebra acting on a Hilbert space $H$ are trivial when the coefficients lie in any ultraweakly closed subalgebra of $B(H)$ which contains the nest algebra, and in [4] Lance gave another proof of this result as part of a wider cohomology result. The extension of this result on Banach spaces was first considered by Spivack [5], who proved that the first cohomology group $H^{1}(\operatorname{alg} \mathcal{N}, B(X))=0$, where $X$ is a Banach space, $B(X)$ is the algebra of all linear bounded operators on $X$, and $\mathcal{N}$ is a uniformly bounded projection nest on $X$. In [2] the author extended Lance's result (for any order cohomology groups) to any closed subspace nest on reflexive Banach spaces.

The purpose of this short note is to prove that the first cohomology group is trivial when the nest is any closed subspace lattice and the coefficients lie in any weakly closed bimodule of $B(X)$ which contains the nest algebra, where $X$ is any normed space.

Let $X$ be a fixed normed space. A nest $\mathcal{N}$ in $X$ is a family of closed subspaces of $X$ that is totally ordered by inclusion and contains (0) and $X$ and the join (closed linear span) and meet (intersection). The associated nest algebra is alg $\mathcal{N}=\{T \in B(X): T E \subseteq E$ for every $E$ in $\mathscr{N}\}$.

Recall that a derivation on an algebra $A$ in $B(X)$ is a linear map $\delta$ from $A$ into $M(M$ is a bimodule of $A$ in $B(X))$ such that $\delta(S T)=S \delta(T)+\delta(S) T$ for all $S, T$ in $A . \delta$ is inner if it is of the form $\delta(S)=T S-S T$ for all $S$ in $A$, for some $T$ in $M$. We denote by $\partial T$ the derivation which is implemented by $T$. For the definition and notation of cohomology groups we refer to [4]. It is known that the first cohomology group $H^{1}(A, M)=0$ if and only if every continuous derivation is inner.

Let $\mathcal{N}$ be a nest on $X$ and $E \in \mathscr{N}, E \neq 0$ and denote by $E_{-}$the element $\bigvee\{F \in \mathscr{N}: F<E\}$. It is known that a rank one operator $x \otimes x^{*} \in \operatorname{alg} \mathscr{N}$ if

Received by the editors July 22, 1991 and, in revised form, December 10, 1991.

1991 Mathematics Subject Classification. Primary 47D25, 47D15, 47B47.

Key words and phrases. Cohomology group, nest algebra. 
and only if $x \in E$ and $x^{*} \in E_{-}^{\perp}$ for some $E$ in $\mathcal{N}$, where $x \otimes x^{*}(y)=x^{*}(y) x$ for every $y$ in $X$ and $\left(E_{-}\right)^{\perp}=\left\{f \in X^{*}: f\left(E_{-}\right)=(0)\right\}$.

Lemma 1. Let $E$ be a closed subspace of $X$ and $f \in E^{\perp}$ such that $\|f\|=1$. Then there exists an element $y$ in $X$ such that $\|y\| \leq 2$ and $f(y)=1$.

Proof. Let $x \in X$ such that $\|x\|=1$ and $f(x) \geq \frac{1}{2}$, let $y=x / f(x)$. Then $\|y\| \leq 2$ and $f(y)=1$.

Lemma 2. Let $\mathcal{N}$ be a nest on $X$ and $E \in \mathscr{N}$. If $T \in B(E, X)$ such that $T S=S T$ on $E$ for all $S$ in alg $\mathcal{N}$, then $T=\lambda I$ on $E$ for some $\lambda$ in $\mathbb{C}$.

Proof. For every $x$ in $E$, we complete the proof by showing that $T x=\lambda_{x} x$ for some $\lambda_{x}$ in $\mathbb{C}$.

Let $E_{x}=\bigwedge\{F: x \in F$ and $F \in \mathscr{N}\}, x \neq 0$.

(i) If $\left(E_{x}\right)_{-}<E_{x}$, we may take $f \in\left(E_{x}\right)_{-}^{\perp}$ such that $f(x)=1$; thus, by $T(x \otimes f) x=(x \otimes f) T x$, we have $T x=f(T x) x$.

(ii) Suppose that $\left(E_{x}\right)_{-}=E_{x}$. Then there exist $E_{n}$ in $\mathcal{N}$ and $x_{n}$ in $E_{n}$ such that $E_{n}<E$ and $x_{n} \rightarrow x$. Take $f_{n} \in\left(E_{n}\right)_{-}^{\perp}$ such that $f_{n}(x)=1$. Then $x_{n} \otimes f_{n} \in \operatorname{alg} \mathcal{N}$, and thus $T\left(x_{n} \otimes f_{n}\right) x=\left(x_{n} \otimes f_{n}\right) T x$, i.e., $T x_{n}=f_{n}(T x) x_{n}$. Let $n$ tend to $\infty$. Then we have $T x=\lambda x$ for some $\lambda \in \mathbb{C}$.

From (i) and (ii), we complete the proof.

Theorem 3. Let $\mathcal{N}$ be a nest on $X$. Then $H^{1}(\operatorname{alg} \mathcal{N}, B(X))=0$.

Proof. Let $\delta$ be a continuous derivation from alg $\mathscr{N}$ into $B(X)$.

(1) If $X_{-}<X$, we can take $f \in\left(X_{-}\right)^{\perp}$ and $y \in X$ such that $f(y)=1$. Define $T \in B(X)$ as

$$
T x=\delta(x \otimes f) y, \quad x \in X .
$$

Then it is easy to verify that $\delta=\partial T$.

(2) Suppose that $X_{-}=X$. Then $X=\bigvee E_{\alpha}$, where $\left\{E_{\alpha}\right\}$ is a totally ordered subfamily of $\mathscr{N}$ such that $E_{\alpha}<X$ for each $\alpha$.

For each $\alpha$, by Lemma 1 , we may take $f_{\alpha} \in E_{\alpha}^{\perp}$ and $y_{\alpha} \in X$ such that $\left\|f_{\alpha}\right\|=1, f_{\alpha}\left(y_{\alpha}\right)=1$, and $\left\|y_{\alpha}\right\| \leq 2$. Define $T_{\alpha} \in B\left(E_{\alpha}, X\right)$ as

$$
T_{\alpha} x=\delta\left(x \otimes f_{\alpha}\right) y_{\alpha}, \quad x \in E .
$$

Then $\left\|T_{\alpha}\right\| \leq 2$, and for any $S$ in alg $\mathcal{N}$ and any $x$ in $E_{\alpha}$, we have

$$
\begin{aligned}
T_{\alpha} S x-S T_{\alpha} x & =\delta\left(S x \otimes f_{\alpha}\right) y_{\alpha}-S \delta\left(x \otimes f_{\alpha}\right) y_{\alpha} \\
& =\delta(S)\left(x \otimes f_{\alpha}\right) y_{\alpha}=\delta(S) x ;
\end{aligned}
$$

thus $\delta=\partial T$ on $E_{\alpha}$.

Now for any $\beta>\alpha$ and $S$ in alg $\mathcal{N}, x \in E_{\alpha}$, we have

$$
\begin{aligned}
\left(T_{\alpha}-T_{\beta}\right) S x & =\delta\left(S x \otimes f_{\alpha}\right) y_{\alpha}-\delta\left(S x \otimes f_{\beta}\right) y_{\beta} \\
& =S \delta\left(x \otimes f_{\alpha}\right) y_{\alpha}+\delta(S) x-S \delta\left(x \otimes f_{\beta}\right) y_{\beta}-\delta(S) x \\
& =S\left(T_{\alpha}-T_{\beta}\right) x
\end{aligned}
$$

thus, by Lemma 2 , there exists $\lambda_{\alpha \beta}$ such that $T_{\alpha}-T_{\beta}=\lambda_{\alpha \beta} I$ on $E_{\alpha}$.

Fix $\alpha_{1}$ such that $E_{\alpha_{1}} \neq 0$, and let $T_{\alpha_{1}}=B_{\alpha_{1}}$ and $B_{\alpha}=\lambda_{\alpha_{1} \alpha} I+T_{\alpha}$ for $\alpha>\alpha_{1}$. Then $\delta=\partial B_{\alpha}$ on $E_{\alpha}$ for each $\alpha>\alpha_{1}$. It is not difficult to verify that $B_{\alpha}$ and $B_{\beta}$ agree on $E_{\alpha}$ wyhen $\alpha<\beta$ and $\left\|B_{\alpha}\right\| \leq 6$ for $\alpha>\alpha_{1}$. Thus there 
is a unique operator $T$ in $B(X)$ such that $T x=B x$ whenever $x$ belongs to $E_{\alpha}\left(\alpha>\alpha_{1}\right)$; therefore, $\delta=\partial T$, which completes the proof.

Recall that the weak operator topology on $B(X)$ is the topology determined by the seminorms $\left\{\phi_{x, x^{*}}: x \in X\right.$ and $\left.x^{*} \in X^{*}\right\}$, where $\phi_{x, x^{*}}(T)=\left|x^{*}(T x)\right|$ for every $T$ in $B(X)$. In [2] the author generalized Erdös's density theorem to normed space cases, saying that every nest algebra alg $\mathcal{N}$ on $X$ is generated by its rank one operators in the weak operator topology. Thus, from [3, proof of Proposition 2.2] we have that any weakly operator topology closed left or right module $M$ of $\operatorname{alg} \mathcal{N}$ is reflexive, i.e., $M=\{T \in B(X): T x \in[M x]\}$ for every $x$ in $X,[\cdot]$ denotes the norm closure. Therefore, by [3, Corollary 3.7], $C(\operatorname{alg} \mathcal{N}, M)=M$ if $M$ is a weakly operator topology closed bimodule of $\operatorname{alg} \mathscr{N}$ which contains alg $\mathscr{N}$, where $C(\operatorname{alg} \mathcal{N}, M)=\{T \in B(X): T A-A T \in$ $M$ for every $A$ in alg $\mathscr{N}\}$. Combining this result and Theorem 3, we obtain

Corollary 4. Let $\mathcal{N}$ be a nest on $X$. Then $H^{1}(\operatorname{alg} \mathcal{N}, M)=0$ for any weakly operator topology closed bimodule $M$ of alg $\mathcal{N}$ which contains the nest algebra.

\section{REFERENCES}

1. E. Christensen, Derivations of nest algebras, Math. Ann. 229 (1977), 155-161.

2. Han Deguang, Cohomology of certain reflexive operator algebras, J. Operator Theory (to appear).

3. __ Rank one operators and bimodules of reflexive operator algebras in Banach spaces, $\mathrm{J}$. Math. Anal. Appl. 161 (1991), 188-193.

4. E. C. Lance, Cohomology and perturbations of nest algebras, Proc. London Math. Soc. (3) 43 (1981), 334-356.

5. M. Spivack, Derivations and nest algebras on Banach spaces, Israel J. Math. 50 (1985), 193-200.

Department of Mathematics, Qufu Normal University, Qufu, 273165, Shandong, PeOple's RePublic of China 